\title{
LA TEORÍA MWC (MONOD, WYMAN Y CHANGEUX): EL SISTEMA ALOSTÉRICO +
}

\author{
K. Alleva*, J.A. DíeZ** y L. Federico*** \\ Universidad de Buenos Aires (Argentina) / Universidad de Barcelona, \\ LOGOS Research Group (España) / Universidad Nacional de Quilmes / \\ Universidad Nacional de Tres de Febrero / CONICET (Argentina)
}

\section{Resumen}

El objetivo del presente trabajo es presentar los primeros pasos de una reconstrucción estructuralista de la teoría alostérica MWC (Monod, Wyman y Changeux). Esta teoría fue presentada en dos artículos publicados en Journal of Molecular Biology, en el primero de ellos "Allosteric proteins and cellular control systems", de 1963, una versión simplificada, y en el segundo, "On the nature of allosteric transitions: a plausible model", publicado en la misma revista en 1965, una versión más acabada. En estos trabajos, el ganador del premio Nobel en Fisiología, Jacques Monod, introduce por primera vez un nuevo concepto que revolucionará la bioquímica y biología de la época: el alosterismo.

Palabras claves: Alosterismo, Teoría MWC, bioquímica, estructuralismo

\section{Abstract}

The aim of this paper is to present the first steps of a structuralist reconstruction of MWC (Monod, Wyman and Changeux') allosteric theory. This theory was presented in two articles published in Journal of Molecular Biology, in the first one, "Alosteric proteins and

Recibido: 11/03/2011. Aceptado: 16/05/2012.

+ Este artículo fue realizado con la ayuda de los proyectos de investigación PICTR2006 No 2007 y PICT2007 No 1558 de la Agencia Nacional de Promoción Científica y Tecnológica (Argentina), PIP0190 y PIP N 112-201101-01135 de CONICET (Argentina) y FFI200801580/CONSOLIDER INGENIO CSD2009-0056 del Ministerio de Ciencia e Innovación (España).

* Universidad de Buenos Aires. e-mail: karina.alleva@gmail.com

** Universidad de Barcelona, LOGOS Research Group. e-mail: jose.diez@ub.edu

*** Universidad Nacional de Quilmes / Universidad Nacional de Tres de Febrero / CONICET. e-mail: luciafed@hotmail.com 
cellular control systems" (1963), a simplified version, and in the second one, "On the nature of allosteric transitions: a plausible model", published in the same journal in 1965, in a more complete version. In these articles, the winner of the Nobel Prize in Physiology Jacques Monod introduces for the first time a new concept that will revolutionize the biochemistry and biology of the time: allosterism.

Keywords: Allosterism, MWC Theory, biochemestry, structuralism

\section{Introducción}

Creo que he descubierto el segundo secreto de la vida... Créase o no, el rol regulatorio de las proteínas alostéricas es absolutamente fundamental, explica todo: la acción hormonal, la función del represor, la cinética enzimática no-michaelianas...

Jaques Monod (1961)

En 1965 Monod recibiría, junto a François Jacob y André Lwoff, el Premio Nobel de Fisiología por su confirmación de la hipótesis del "Operon LAC”, hipótesis que explica la regulación de la expresión génica de las proteínas. Monod propuso la existencia de un regulador (el represor mencionado en la cita, molécula que intuía se trataba de una proteína) que al unirse al ADN era capaz de controlar la síntesis proteica. Si bien estos estudios están claramente centrados en la regulación de la actividad de las proteínas a largo plazo, Monod también se dedicó al estudio de la actividad biológica de las enzimas ${ }^{1}$ y su regulación rápida. En los años `60 los patrones de actividad biológica no podían ser explicados por las teorías del momento, como la enzimología o la cinética enzimática. Si bien el Nobel recibido no se debió a estas investigaciones, tanta relevancia tenían que en las últimas páginas de la lectura que realizó al recibir el premio se tomó el tiempo para exponer el por él llamado "modelo de interacción alostérica" o "modelo alostérico":

La "invención" de las interacciones alostéricas indirectas, que dependen exclusivamente de la propia estructura de la proteína, esto es de su código genético, habría liberado a la evolución molecular de sus limitaciones (Monod, 1965, p. 204).

Monod consideró desde un primer momento que la regulación alostérica era absolutamente fundamental para explicar muy diversas cuestiones metabólicas. Este modelo fue presentado en dos artículos publicados en Journal of Molecular Biology, el primero de ellos "Allosteric proteins and

${ }^{1}$ Las enzimas son moléculas proteicas que catalizan las trasformaciones químicas que ocurren en los organismos vivos. Las enzimas presentan al menos un sitio de unión específico, el sitio activo, para unirse a la sustancia que será químicamente transformada, el sustrato, para que se produzca su transformación. 
cellular control systems" en 1963 (Monod, Changeux \& Jacob), en una versión simplificada, y el segundo, una versión más acabada, "On the nature of allosteric transitions: a plausible model" publicado en la misma revista en 1965 (Monod, Wyman \& Changeux).

Haciendo un aporte más al entendimiento metateórico de la bioquímica y la biología, el objetivo que nos proponemos en este trabajo es efectuar los primeros pasos de una reconstrucción estructuralista de la teoría alostérica (posteriormente llamada "modelo alostérico concertado" o "modelo MWC") tal como fuera presentada en la publicación del año $1965 .^{2}$ Se trata de la primera reconstrucción de la estructura de una teoría en al ámbito de la bioquímica alostérica, un campo muy reciente de la bioquímica que aún está en evolución, con algunos elementos parcialmente confusos que nuestra reconstrucción ayuda a clarificar.

\section{La Teoría MWC}

Desde el comienzo de los estudios bioquímicos, la mayor parte de las curvas conocidas como dosis-respuesta, ${ }^{3}$ con las que se representa la actividad biológica de las proteínas, tenían perfil hiperbólico. Sin embargo, muchas otras proteínas presentaban en lugar de éste uno sigmoideo o también llamado "cooperativo" (Fig. 1).

A partir de 1910 comenzaron a proponerse diferentes teorías que intentaban dar cuenta de este tipo de perfiles de actividad de proteínas biológicamente importantes, como por ejemplo el de la hemoglobina. Esta molécula, encargada de transportar oxígeno en los glóbulos rojos, esta formada por cuatro subunidades. Cada subunidad recibe el nombre de protómero, y la proteína como un todo se denomína oligómero. Cada protómero consta a la vez de un sitio de unión para el sustrato (o molécula sujeto de la acción de la proteína) en este caso el oxígeno. En presencia de cierta cantidad de

2 Si bien en 1961 Monod y Jacob utilizan por primera vez el termino "alostérico" para dar cuenta de la actividad atípica de algunas enzimas, en los años siguientes este concepto se fue modificando hasta consolidarse en 1965. Actualmente el término se aplica a aquellas proteínas formadas por más de una subunidad que sufren modificaciones en su estructura o estado conformacional.

${ }^{3}$ Curva dosis-respuesta remite a un clásico modo de representar la actividad biológica de muchas proteínas. Este tipo de representación corresponde a graficar datos experimentales como respuesta biológica obtenida en función de la cantidad de un sustrato en un volumen fijo o, lo que es lo mismo, concentración, agregado a una cantidad determinada de proteína ensayada. 


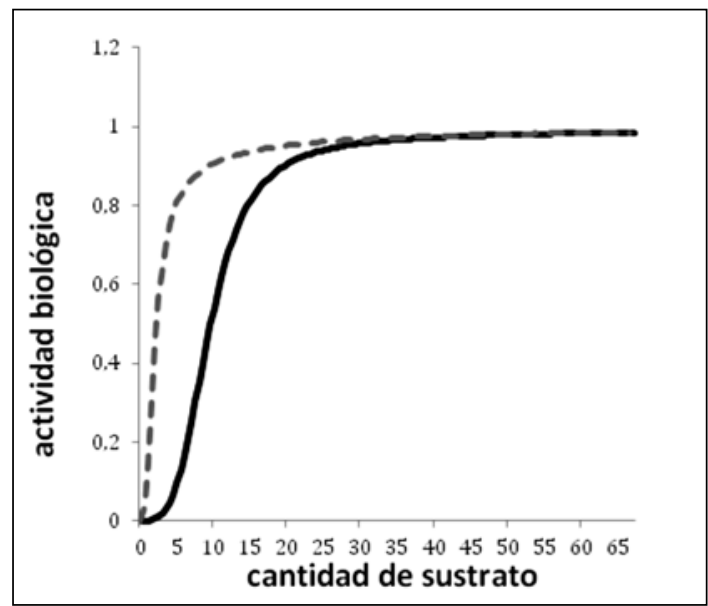

Figura 1. La figura muestra los perfiles hiperbólicos (líneas punteada y gris) y sigmoideo (línea llena negra) que puede adquirir una curva experimental dosis-respuesta representando la actividad biológica en función de cantidad de sustrato.

oxígeno la hemoglobina tiene la capacidad de unir una molécula de este gas en cada uno de sus cuatros sitios de unión para su transporte. Midiendo el porcentaje de oxígeno unido a la hemoglobina en función de la cantidad de oxígeno en el medio, se obtiene su curva de actividad, una manera en que se cuantifica la actividad biológica. Así, la actividad de un oligómero, consiste en la realización de cierta función cuando cierta sustancia llamada "sustrato" se une a alguno de los sitios de unión.

Casi medio siglo después de comenzados los estudios sobre el patrón de actividad de la hemoglobina se descubrió que algunas de las proteínas funcionales conocidas como enzimas también presentaban curvas dosis-respuesta cooperativas. Este es el caso de la fosfofructoquinasa (PKF), una enzima cuya función en la célula es la de catalizar la transformación química de la fructosa-6-fosfato, su sustrato, a fructuosa-1-6-bifosfato. La actividad de esta y otras enzimas, como la desoxitimidina kinasa que retomaremos más adelante, no podían explicarse mediante el uso de la teoría vigente, la cinética enzimática michaeliana. Dicha teoría daba cuenta solamente de curvas no cooperativas. Durante estos años en el que el cooperativismo empezaba a desarrollarse teóricamente, se halló que no solo algunas enzimas oligoméricas presentaban esta peculiaridad sino que su actividad biológica podía ser modificada, aumentada o disminuida, por sustancias específicas llamadas 'reguladores', ("activadores" e "inhibidores" respectivamente). 
Por ejemplo, la enzima PKF es activada por la sustancia adenosinamonofosfato (AMP) e inhibida por otra sustancia, el citrato. Estudios estructurales posteriores mostraron que cada subunidad del oligómero presentaba un sitio de unión específico para cada tipo de regulador sea activador o inhibidor, además de aquel para el sustrato.

Si bien la búsqueda de una explicación para este patrón de actividad sigmoideo comenzó en la primera década del siglo XX, recién en 1965 una de esas propuestas se convirtió en un hito en la historia de la bioquímica. Fue en este año cuando se presentó, en su forma más completa, la teoría alostérica. Esta teoría, que recibió el nombre de "modelo MWC", por sus autores Monod, Wyman y Changeux, pretendía dar cuenta de un mecanismo molecular, el alosterismo, que explicara estas correlaciones entre el cambio en la actividad biológica y la presencia de diferentes sustancias o ligandos, ${ }^{4}$ (sustrato, activadores e inhibidores) de la siguiente manera:

- le atribuye a los oligómeros dos estados conformacionales (es decir, dos estados diferentes de simetría estructural $)^{5}$ en la que los oligómeros pueden tener más o menos afinidad con respecto a los ligandos,

- y postula algunas conexiones nomológicas entre los estados conformacionales, las afinidades, los estados de unión a los ligandos y la actividad.

La mayor novedad de este modelo son, por tanto, los dos estados conformacionales postulados como existentes para un mismo oligómero, teniendo cada uno diferente actividad biológica: el estado "relajado" (R) con alta actividad biológica y el estado "tenso" (T) con baja actividad.

En ausencia de ligando se asume que, para cada población dada, los estados $\mathrm{T}$ y $\mathrm{R}$ están en un equilibrio, esto significa que la cantidad total de oligómeros en estado $\mathrm{T}$ y la cantidad total de oligómeros en estado $\mathrm{R}$ son constantes en todo momento a pesar de que cada oligómero en sí mismo

${ }^{4}$ Se habla generalmente de ligando, para hacer referencia a la molécula capaz de ligarse o unirse a un sitio determinado de la proteína estudiada que desencadena o modifica la actividad biológica de la misma

${ }^{5}$ Las proteínas son moléculas complejas, formadas por una cadena de aminoácidos (conocida como estructura primaria), que se pliega de distinta manera generando dominios estructurales (adquiriendo lo que se conoce como estructura secundaria) que a su vez se organizan en general dando una especie de estrucutra tridimensional en forma de ovillo (llamada estructura terciaria). Si la proteína está formada por varias subunidades que se asocian, cada una con su estructura terciaria, adquiere lo que se conoce como estructura cuaternaria. A partir de la naturaleza y forma de estas subunidades se traza la simetría. La forma funcional se adquirirá en la estructura terciaria si la proteína es monomérica o en la cuaternaria si el oligomérica. 
este continuamente cambiando de estado. Dado que la cantidad de oligómeros en cada estado es constante, la relación entre ellas también lo es, y el valor de dicha relación es el parámetro conocido como "constante de equilibrio", en este caso en particular la constante de equilibrio recibe el nombre de "constante alostérica" $\left(\mathbf{t}_{0}\right)$ y al cambio de un estado a otro se lo llama "transición alostérica".

La unión del ligando a cada sitio específico del protómero, ya sea en la conformación $\mathrm{R}$ o $\mathrm{T}$, alcanza también un equilibrio; las constantes de equilibrio correspondientes a los equilibrios de unión de cada ligando en cada estado conformaciónal reciben el nombre de "constantes microscópicas de disociación”. Dichas constantes son el parámetro que indica cuánta afinidad $^{10}$ tiene el sitio de unión del protómero por el ligando en una conformación dada. Así el modelo tiene en cuenta las constantes microscópicas de disociación para los activadores e inhibidores y supone que el activador tiene más afinidad por la proteína en estado conformacional $\mathrm{R}$, mientras que el inhibidor la tiene por la conformación T (Fig 2).
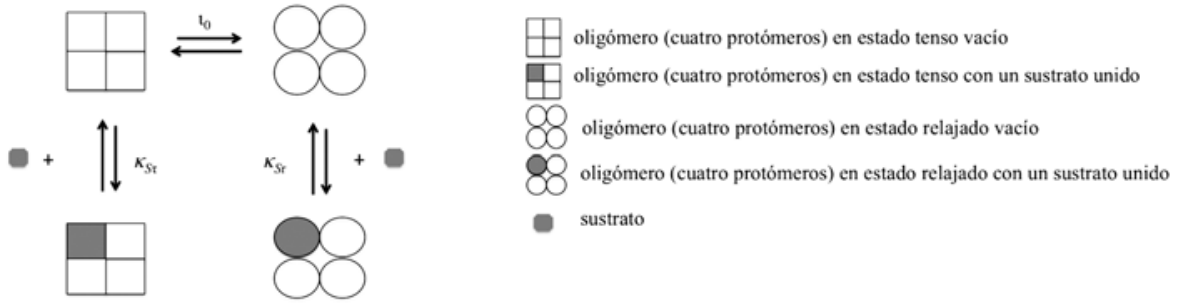

Figura 2. En este esquema se representa la transición alostérica de un oligómero formado por cuatro protómeros y sus equilibrios de unión a sustrato. Los cuadrados indican protómeros en estado tenso, mientras que los cículos señalan protómeros en estado relajado. En la parte superior de la figura se muestra el equilibrio del oligómero vació pasando de un estado conformacional a otro regido por la constnate alostérica $\left(\mathbf{t}_{0}\right)$. Los laterales del esquema muestran los equilibrios de unión de un sustrato a uno de los sitios de unión de un protómero del oligómero, estos equilibrios se hallan regidos por sus correspondientes constantes $k_{s t}$ $\mathrm{o} k_{\mathrm{Sr}}$. El sustrato puede seguir uniéndose hasta completar todos los sitios disponibles para él.

${ }^{6}$ Las constantes de disociación, como toda constante de equilibrio, son un parámetro que relaciona la cantidad de las especies moleculares que participan de dicho equilibrio. Un equilibrio de este tipo se puede representar con la siguiente ecuación química: $\mathrm{A}+\mathrm{B} \leftrightarrow \mathrm{AB}$, donde del lado izquierdo de la ecuación se muestra que A y B están disociados y del lado derecho se indica que A y $\mathrm{B}$ se han unido. El valor de la constante de este equilibro es igual al resultado de dividir la concentración de $\mathrm{AB}$ ( $\mathrm{A}$ unido a $\mathrm{B}$ ) por el producto de concentración de A por la concentración de $\mathrm{B}$, dicho en forma matemática $[\mathrm{AB}] /[\mathrm{A}] .[\mathrm{B}]=$ Cte. Este parámetro mide la fuerza o "afinidad" de la unión de A con B. 
Una característica fuerte del modelo MWC, es la necesaria simetría del oligómero. Esto es, un oligómero está en estado R si todos sus protómeros están en estado $\mathrm{R}$, y esta en estado $\mathrm{T}$ si todos sus protómeros están en estado $\mathrm{T}$, ya que no existe la posibilidad de que un oligómero tenga algunos protómeros en estado $\mathrm{R}$ y otros en estado T. Así, cuando un oligómero pasa de un estado a otro, todos sus protómeros también lo hacen. Por eso a este modelo se lo llamó "concertado", diferenciándolo de otro modelo, que surgió como respuesta al modelo de Monod: el Modelo KNF o "secuencial" (Kosland, Némethy y Filmer, 1966) que sí permitía estados híbridos (un oligómero con algunos de sus protómeros en $\mathrm{R}$ y otros en $\mathrm{T}$ ).

Hasta aquí el modelo MWC plantea las siguientes hipótesis sobre la estructura de las proteínas alostéricas (Monod, Wyman y Changeux, 1965, pp.89-90):

i. las proteínas alostéricas son "oligómeros" formados por subunidades idénticas llamadas "protómeros";

ii. los protómeros se asocian de manera tal que la molécula posee un eje de simetría;

iii. al ligando le corresponde un sitio de unión específico en el protómero;

iv. los oligómeros pueden adoptar dos estados conformacionales diferentes; estos estados, que difieren por su actividad biológica, se encuentran, en ausencia de ligando, en equilibro;

v. la conformación de cada protómero está restringida por la de los otros protómeros para mantener la simetría del oligómero;

vi. la afinidad de los ligandos por el sitio de unión específico es diferente para cada estado conformacional; y

vii. aun cuando el oligómero este en uno u otro estado, la simetría se conserva.

Otra de las características principales de este primer modelo alostérico es que no se trata de un modelo cíclico. Sólo las formas no unidas a ligando, tanto del estado tenso no unido $\left(\mathrm{T}_{0}\right)$ como del estado relajado no unido $\left(\mathrm{R}_{0}\right)$, pueden cambiar a la otra conformación. Una vez unida la proteína a un ligando en alguna de estas conformaciones, ésta queda "fijada" de manera tal que no puede producirse una nueva transición alostérica. En palabras de Bindslev, quien ha realizado uno de los análisis más exhaustivos sobre el alosterismo,

la omisión de una reacción cíclica no fue explicitada, sin embargo aparece en los esquemas de reacción y las formulaciones presentadas en el trabajo de 1965. El punto omitido, un equilibrio reverso, o aún sólo un equilibrio entre estados ligados, no estaba permitido [...]. De acuerdo con esto, el modelo MWC debería haber sido designado 'modelo de un solo equilibrio no-ligado'. [...]. La constricción de un único 
equilibrio en el estado no ligado planteada por el modelo MWC es una de las ventajas más fuertes del mismo ya que reduce la complejidad del sistema concertado a sólo cuatro parámetros para su descripción, la constante alostérica, las constantes de disociación del estado T y R y el número de sitios de unión (2008, p. 381).

A su vez, el modelo distingue dos tipos de interacciones entre oligómero y ligandos que conllevan a dos efectos diferentes: la interacción del oligómero con ligandos iguales, lo que los autores del modelo han denominado "efecto homotrópico", y la interacción con ligandos diferentes, llamada "efecto heterotrópico". El primer efecto permite explicar la "cooperatividad" positiva ejercida en la proteína por cada unión del ligando a cada uno de los sitios del oligómero. Según el modelo, cuando un oligómero que está en equilibrio R-T (sin ligando unido) une una molécula de ligando a uno de sus protómeros en estado conformacional $\mathrm{R}$, se estabiliza de manera tal que permanece más tiempo en ese estado, generandose un desplazamiento del equilibrio R-T hacia R. Esto repercute en un aumento de la actividad biológica. El segundo caso permite explicar el cambio en los patrones de actividad (aumento o disminución), provocado por activadores o inhibidores (llamados también "moduladores" o "efectores"). A su vez el modelo también permite predecir la actividad biológica de las proteínas no alostéricas, como el de las enzimas con cinética del tipo Michaelis-Menten, punto que trataremos en las especializaciones.

Es interesante destacar que si bien esta teoría fue presentada para dar cuenta de la actividad biológica de algunas proteínas, en realidad lo que predice no es exactamente eso, sino dos parámetros de alguna manera vinculados a esta actividad: la "función de saturación" $(\bar{\gamma})$ o fracción de sitios unidos con el ligando (para toda conformación), y la "función de estado $\mathrm{R}$ ” $(\bar{R})$ o fracción de proteínas en estado conformacional R. Ambos parámetros están estrechamente ligados entre sí: el modelo MWC asume que la actividad biológica se puede analogar cualitativametne a la función de saturación.

Según nuestro punto de vista, compartido por otros autores (p.e. Bindslev 2008), para representar la actividad biológica de los oligómeros es más adecuada la función de estado $\bar{R}$, ya que ésta considera la fracción de estados relajados que son los responsables de la actividad del oligómero. Por otro lado, $\bar{R}$ es capaz de capturar la actividad biológica espontánea que pudieran tener los oligómeros ${ }^{7}$. Sin embargo, esta función no fue considerada por los autores del modelo para dar cuenta de la actividad biológica de los sistemas estudiados. Si bien en aquel trabajo no se avanzó conceptualmente en cuál

${ }^{7}$ La actividad espontánea es aquella que ocurre en ausencia de ligando y se da solo para ciertos tipos de oligómeros alostéricos como ser cierta clase de proteínas llamadas canales. 
era la relación entre actividad biológica y la fracción de oligómeros ligados (medido por la función de saturación) o la fracción de oligómeros en estado $\mathrm{R}$ (medido por la función de estado), se asumió en aquél momento que sería la función de saturación la que podría analogarse, cualitativamente al menos, a la actividad biológica de la proteína. Creemos que esto pudo deberse a que las aplicaciones intencionales del momento, los sistemas alostéricos concretos a que se pretendía aplicar el modelo, comprendían las enzimas y la hemoglobina. Las enzimas no tienen actividad espontánea, esto es, no presentan actividad biológica en ausencia de ligando, y la hemoglobina es una molécula para la cual el mero ligado ha sido considerado su actividad biológica, por ello si no está unida a ligando alguno no se considera activa. Ahora bien, años después de presentado el modelo MWC se comprobó que existían proteínas oligoméricas, como los llamados “canales", que presentaban actividad espontánea. ${ }^{8}$ Para esos oligómeros las predicciones sobre la actividad no pueden en ningún caso adecuarse a la función de saturación, ya que ésta no considera al estado relajado sin ligando unido $\left(\mathrm{R}_{0}\right)$ ahora portador de actividad. Esto es, si bien los autores decidieron analogar cualitativamente la curva de actividad biológica a la función de saturación, estrictamente, la función de saturación no explica la actividad biológica sino que explica cómo evolucionarán las uniones ligando-oligómero en el tiempo. Sin embargo, aunque los autores no lo expliciten, al utilizar en el trabajo de 1965 la función de saturación para este tipo de proteínas (aquellas sin actividad en el estado $\mathrm{R}_{0}$ ) y las restricciones experimentales consideradas (el valor que toma la constante alostérica y la afinidad del ligando por el estado R), están haciendo uso de una ecuación homologable a la función de estado $\bar{R}$, dado que en esas condiciones se elimina una posible actividad espontánea. Este uso de la función de saturación como cualitativamente representativa de la actividad biológica por parte de Monod y colaboradores ha sido, por lo antes mencionado, objeto de críticas y de intentos de superación, pues fue considerado algunas veces como una aparente confusión, y sigue siendo una cuestión abierta. Una virtud de nuestra reconstrucción es que clarifica por qué es la función de estado y no la de saturación la que interviene en la formulación de las leyes.

Dado que al momento de ser presentada la teoría esta no pretendía ser aplicada a estas proteínas, el caso posible de la actividad espontánea no fue considerada.

${ }^{8}$ En el caso de la mayoría de los canales la actividad se mide como corriente eléctrica. Los ligandos sustratos son moléculas distintas de aquellas que atraviesan el canal y si bien en ausencia de ligando se detecta actividad, en presencia de estos la actividad se puede disparar de manera alostérica. 
La fuerza que tuvo y sigue teniendo el modelo MWC radica en haber sido la primera teoría en intentar dar cuenta de las propiedades funcionales de las proteínas regulatorias, en su mayoría oligoméricas, a partir de sus características estructurales, y en un mismo paso, ensayar una respuesta a la pregunta de por qué en la evolución molecular han sido favorecidas este tipo de proteínas grandes y formadas por subunidades. La razón de esta selección positiva de estructuras oligoméricas altamente simétricas, según los autores, podría deberse a la cooperatividad inherente a este tipo de estructuras. La forma sigmoidea de los patrones de actividad biológica de los sistemas alostéricos daría la posibilidad de que la regulación metabólica posea valores umbrales a partir de los cuales una respuesta biológica pueda ser disparada.

Hoy día el modelo alostérico MWC sigue siendo central en la bioquímica, biología y ciencias relacionadas como las biomédicas, en la formación de las nuevas generaciones de científicos en estas disciplinas, aunque por sus carencias ya son varias las teorías alternativas que han tomado su espíritu generando modelos más sofisticados que puedan dar cuenta con mayor precisión de los resultados experimentales. Sin embargo, el modelo MWC fue una teoría central para el comienzo de los estudios de las relaciones estructura-función de proteínas que actualmente aborda, en general, la biofísica. En efecto, la teoría alostérica ha sido considerada por Darden y Maull (1977) como un ejemplo relevante de teoría de "intercampos", en este caso, una teoría que se origina a partir de la interacción de dos campos el de la bioquímica y el de la físicoquímica de la época. Según Monod y colaboradores, esta teoría debe considerarse como una gran guía heurística para la investigación bioquímica y biológica, ya que, en palabra de los propios autores, el valor del modelo radica en haber presentado:

una relación simple general e inicial entre simetría y función [que] puede explicar la emergencia, evolución y propiedad de las proteínas oligoméricas (Monod et al., 1965, p117).

\section{El elemento teórico básico}

\subsection{El núcleo teórico básico de la teoría MWC}

Antes de iniciar la reconstrucción, recordamos muy brevemente las principales nociones estructuralistas. ${ }^{9}$ El tipo más simple de estructura conjun-

${ }^{9}$ Este trabajo presupondrá familiaridad por parte del lector con dicha concepción. Se recomienda consultar Balzer, Moulines \& Sneed (1987) para una presentación completa, 
tista susceptible de ser considerada una reconstrucción formal del concepto intuitivo de teoría científica es el denominado elemento teórico $\mathrm{T}$ y puede ser identificado, en una primera aproximación, con el par ordenado consistente en un núcleo teórico $\mathbf{K}$ y en un conjunto de aplicaciones pretendidas, propuestas o intencionales $\mathbf{I}: \mathrm{T}=\langle\mathbf{K}, \mathbf{I}\rangle$. El núcleo $\mathbf{K}$ es la parte formal de la teoría, que expresa los recursos conceptuales a diferentes niveles y las restricciones-leyes que según la teoría rigen su ámbito de estudio. El conjunto de aplicaciones intencionales I constituye la parte aplicativa del elemento teórico, y especifica, en términos T-no teóricos respecto de la teoría (e.e. pre-teóricos a la teoría T), los sistemas empíricos a los que la teoría pretende aplicarse, de los que se pretende que son regidos por sus restriccionesleyes. El núcleo $\mathrm{K}=\left\langle\mathbf{M}_{\mathrm{p}}, \mathbf{M}, \mathbf{M}_{\mathrm{pp}}, \mathrm{C}, \mathrm{L}\right\rangle$ está constituido por los conjuntos: $\mathrm{M}_{\mathrm{p}}$ de modelos potenciales, que representa el aparato conceptual completo (T-Teórico y T-no teórico) de la teoría, son las estructuras con el tipo de constituyentes adecuado de las que tiene sentido preguntarse si cumplen o no las leyes; $\mathrm{M}$ de modelos actuales o efectivos, que son aquellos modelos potenciales que satisfacen además las leyes de la teoría; $M_{p p}$ de modelos parciales, que son el resultado de "recortar" de los modelos potenciales sus constituyentes T-teóricos y representan pues el aparato conceptual preteórico con el que se describen los fenómenos a explicar (p.e. en mecánica, descripciones cinemáticas de trayectorias explicadas después introduciendo entidades teóricas dinámicas como masas y fuerzas). Por mor de simplicidad, las ligaduras C o constricciones cruzadas, y los vínculos interteóricos L, que representan constricciones nómicas derivadas de la relación con otras teorías no serán analizadas en esta reconstrucción.

\subsubsection{Los modelos potenciales de la teoría MWC}

El conjunto de los modelos potenciales $\mathbf{M}_{\mathbf{p}}$ (MWC) simboliza la clase total de entidades que satisfacen las condiciones estructurales (los axiomas impropios) que caracterizan al aparato conceptual de la teoría y son aquellas estructuras de las cuales tiene sentido preguntarse si son modelos actuales de la teoría, pero que todavía no se sabe si efectivamente lo son.

o Díez \& Lorenzano (2002) para una presentación breve de esta concepción metateórica. Los motivos por los cuales se elige esta concepción se encuentran resumidos en el siguiente diagnóstico de Nancy Cartwright: "Los estructuralistas [...] indudablemente ofrecen el tratamiento más satisfactoriamente detallado y bien ilustrado de la estructura de las teorías científicas disponible" (Cartwright, 2008, p. 65). 
Definición 1. $\mathrm{M}_{\mathrm{p}}(\mathrm{MWC}): \quad x=\left\langle\mathrm{O}, S, A, I, T,\{\tau, \mathrm{r}\}, \rho, \sigma, \beta, \delta, \zeta, \kappa_{S}, \kappa_{A}\right.$, $\left.k_{\mathrm{I}}\right\rangle$ es un sistema MWC potencial $\left(x \in \mathbf{M}_{\mathrm{p}}(\mathbf{M W C})\right.$ ) si y solo si,

(1) O es un conjunto finito, no vacío ("población de oligómeros")

(2) $S, A, I$ son conjuntos finitos no vacíos $\& S \cap I=S \cap A=A \cap I=\varnothing$ ("ligandos")

(3) $T$ es un orden lineal discreto ("tiempo")

(4) $\{\tau, r\}$ es conjunto finito formado por dos elementos ("estado conformacional")

(5) $\rho: \mathrm{O} \rightarrow \mathrm{N}$ es una función (" $\mathrm{n}^{\mathrm{o}}$ de protómeros")

$\rho$ es una función constante: $\forall o, o^{\prime} \in O \rho(o)=\rho\left(o^{\prime}\right)$

$\operatorname{DfAux} 1: \rho(O)=n$ syss $_{\mathrm{df}} \exists o \in O \rho(o)=n$

(6) $\beta=\left\{\left\langle o,\langle s, a, i\rangle_{1}, \ldots,\langle s, a, i\rangle \rho_{(O)}\right\rangle / \forall j:\langle s, a, i\rangle_{j} \in(S \times A \times I) \cup(S \times A \times\{\varnothing\})\right.$ $\cup(S \times\{\varnothing\} \times I) \cup(S \times\{\varnothing\} \times\{\varnothing\}) \cup(\{\varnothing\} \times\{\varnothing\} \times\{\varnothing\})\}[1 \leq j \leq \rho(O)]$ ("unión química oligómero-ligandos")

(7) $\sigma: O \times T \rightarrow \beta$ ("sucesión de uniones del oligómero")

$D f A u x$ 2: $\sigma(o, t)_{j}=$ el $j$-ésimo componente de $\sigma(o, t)[1 \leq j \leq \rho(\mathrm{O})]$

(8) $\delta: T \rightarrow \mathbf{R}^{+}$("actividad biológica")

(9) $\zeta: \beta \times T \rightarrow\{\tau, r\}$ ("estado conformacional“)

$(10) \kappa_{S}:\{\tau, r\} \rightarrow \mathbf{R}^{+}$

$\kappa_{A}:\{\tau, r\} \rightarrow \mathbf{R}^{+}$

$\kappa_{I}:\{\tau, \mathbf{r}\} \rightarrow \mathbf{R}^{+}$(“constantes de disociación”)

tal que

$\left.\forall o, o^{\prime} \in O: \operatorname{si}_{\Psi}\left(\zeta\left(\left\langle o,\langle s, a, i\rangle_{1}, \ldots,\langle s, a, i\rangle \rho_{(O)}\right\rangle, t\right\rangle\right)\right)=\kappa_{\Psi}\left(\zeta\left(\left\langle o^{\prime},\langle s, a\right.\right.\right.$, $\left.\left.\left.\left.i\rangle{ }_{1}, \ldots,\langle s, a, i\rangle^{\prime} r_{(O)}\right\rangle, t^{\prime}\right\rangle\right)\right)$ entonces $\left.\left.\xi\left(\left\langle o,\langle s, a, i\rangle_{1}, \ldots,\langle s, a, i\rangle \rho_{(\mathrm{O})}\right\rangle, t\right\rangle\right)\right)=$ $\left.\xi\left(\left\langle O^{\prime},\langle s, a, i\rangle_{1}, \ldots,\langle s, a, i\rangle^{\prime} r_{(\mathrm{O})}\right\rangle, t^{\prime}\right\rangle\right)$

DfAux 3: $\kappa_{\Psi_{r}}=\kappa_{\Psi}(r)$

$\kappa_{\Psi \tau}=\kappa_{\Psi}(\tau)$

donde $\psi$ es una variable para los diferentes tipos de ligandos $S, A, I$

Aunque hemos indicado entre paréntesis el concepto que expresa cada símbolo, vamos a ver con más detenimiento el significado de los diversos componentes del predicado conjuntista:

(1) O es el conjunto oligómeros y representa a una población de oligómeros. Los oligómeros son proteínas con actividad biológica y están formados por uno o más protómeros. Es un conjunto finito y no vacío.

(2) $S, A, I$ representan los conjuntos de tipos de ligandos. Se caracterizan por ser sustancias que se unen a los oligómeros. $S$ representa el conjunto de los ligandos sustratos que se caracterizan por ser sustancias que al unirse a los oligómeros permiten que estos ejerzan su actividad biológica; $A$ representa el conjunto de los ligandos activa- 
dores, aquellos que al unírseles incrementan la actividad biológica del oligómero; I representa el conjunto de los ligandos inhibidores, aquellos que disminuyen la actividad biológica de los oligómeros al unírseles. Respecto a un tipo de oligómero particular (sea hemoglobina, PKF, etc) no hay sustancias que fueran a la vez sustrato, activador e inhibidor. Por eso la intersección de $S, A$ e $I$ es vacía.

(3) $T$ representa el tiempo. Viene dado por el par $\langle T,<\rangle$, que constituye un orden lineal sobre el conjunto $\mathrm{T}$ de instantes, en donde < representa la relación temporal "es posterior a" (siendo el par $\langle\mathrm{T}$, $<\rangle$ isomórfico con el par $\langle\mathbf{N},<\rangle$, consistente en el conjunto de los números naturales $\mathbf{N}$ y en la relación-menor-que sobre los números naturales).

(4) $\{\tau, r\}$ representa a los dos posibles estados conformacionales que pude adoptar un oligómero, tal que $\tau$ simboliza el estado conformacional tenso y $r$ el estado conformacional relajado.

(5) $\rho$ representa la función número de protómeros. ${ }^{10}$ Los oligómeros están formados por uno o más protómeros, cada uno de los cuales tiene un sitio de unión para cada tipo de ligando (uno para el sustrato, otro para el activador y un tercero para el inhibidor), sitio que se podrá usar o no, e.e. quedar vacío. Puesto que $\rho$ asigna el mismo número de protómeros para todos los oligómeros del sistema (y por tanto representa también el número de sitios de unión) podemos definir entonces "el número de protómeros que tiene los oligómeros del sistema", $\rho(O)$, como constante. ${ }^{11}$

(6) $\beta$ es una relación de unión química o binding entre oligómero y ligandos. Representa todas las posibles combinaciones de estados de unión de los oligómeros del sistema. Cada oligómero o tiene $\rho(o)$ protómeros o sitios de unión para cada tipo de ligando. Sin embargo, en esta teoría, el oligómero no puede unirse a activadores o inhibidores sin unirse a un sustrato. Esto significa que cada protómero tiene la posibilidad de unirse a los tres ligandos: sustrato, activados e inhibidor, o al sustrato y al activador, o al sustrato y al inhibidor o a sólo sustrato o a nada.

${ }^{10}$ Es conveniente aclarar que dado un modelo particular, por ejemplo hemoglobinaoxígeno, todos los oligómeros del mismo tienen el mismo número de protómeros, aunque por supuesto no todos los oligómeros de todos los modelos tienen que tener necesariamente el mismo número de protómeros, pero (5) no lo implica.

${ }^{11}$ La definición auxiliar 1 da cuenta de "el" número de protómeros del los oligómeros (el mismo para cada modelo), definición que luego es útil para los formalismos subsiguientes como por ejemplo el (5). 
(7) o representa la función sucesión de uniones del oligómero. Esta función permite capturar, protómero a protómero, las uniones que se generan entre un oligómero y los distintos tipos de ligandos en el tiempo, y por tanto, cómo estas uniones van variando para un mismo oligómero en cada momento. Si representamos como $\sigma(o, t)$ el estado de unión del oligómero $o$ en el momento $t$, mediante $\sigma(o, t)$ $=\left\langle o,\langle s, a, i\rangle_{1}, \ldots,\langle s, a, i\rangle \rho_{(\mathrm{O})}\right\rangle$ significamos que el oligómero $o$, en el momento $t$ esta unido en el sitio de unión 1 al/los ligando/s tal/es, en el sitio de unión 2 al/los ligando/ tal/es, hasta llegar a los sitios de unión $\rho(O)$.

(8) $\delta$ es una función que representa la actividad biológica. Es un número real positivo que se asigna a la población de oligómeros y representa la "intensidad" de la actividad biológica en un momento dado. Cada tipo de oligómero tiene su propio tipo de actividad, puede ser la transformación de una sustancia en otra (para enzimas), o la unión del ligando para transporte (para la hemoglobina), etc. En el elemento teórico básico la "actividad" se deja genéricamente sin especificar, mientras que se especifica en las diversas especializaciones terminales de este elemento teórico (ver más adelante). Nótese que $\delta$ es un valor "global" de la actividad para una población $O$, este hecho es formalmente expresado mediante una $\delta$ concreta en el modelo que posee como dominio una población $O$ específica. Cuantifica la actividad de una población de oligómeros en el tiempo.

(9) $\zeta$ es la función estado conformacional. Es una función que asigna a un oligómero unido o no a ligandos, uno de los dos estados conformacionales $\tau, r$. Cada oligómero en un estado de unión estará en un tiempo dado en un estado conformacional, y el mismo oligómero en el mismo estado de unión puede estar en diferente estado conformacional en momentos diferentes.

(10) $\kappa_{S}, \kappa_{A}, \kappa_{I}$ son las funciones "constante de disociación" para cada ligando. Cada función representa la afinidad de los oligómeros en un estado conformacional particular por cada ligando. Es, por tanto, la tendencia o predisposición del oligómero para unirse a un ligando en un estado. De ésta se postula que: (i) es la misma para todos los lugares del oligómero correspondientes a un tipo de ligando, por lo que se le puede asignar al oligómero como una unidad, (ii) sólo depende del estado conformacional y (iii) es la misma para todos los oligómeros de la población (es en este sentido que son "constantes”). Por ejemplo ' $\kappa_{A}\left(\zeta\left(\left\langle o,\langle s, a, i\rangle_{1}, \ldots,\langle s, a, i\rangle \rho_{(\mathrm{O})}\right\rangle, t\right)\right)$ ' se lee "la afinidad del oligómero $o$, en el estado de unión ... con un ligando $A$ en el momento $t$ cuyo estado conformacional es $\zeta\left(\left\langle o,\langle s, a, i\rangle_{1}\right.\right.$, 
$\left.\left.\ldots,\langle s, a, i\rangle \rho_{(O)}\right\rangle, t\right)^{\prime}$. Si usamos la variable $\Psi$ para los diferentes tipos de ligando podemos definir ' $\mathrm{K}_{\mathrm{Y}}$ ' $\mathrm{y}$ ' $\mathrm{K}_{\mathrm{Y}}$ ' como abreviaciones para las afinidades de los oligómeros del sistema para el ligando $y$ cuando el oligómero esta en el estado $r, \tau$, respectivamente.

Así, un modelo potencial de MWC es básicamente una serie o sucesión de uniones de un oligómero con sus ligandos, donde este oligómero tiene sitios de unión para dichos ligandos, y es más o menos afín a unirse a estos según esté en estado conformacional tenso o relajado; la población lleva asociada a la vez un tipo y grado de actividad. Estos son los sistemas de los que tiene sentido preguntarse a continuación si cumplen de hecho o no las leyes de MWC que rigen la relación específica entre uniones, estados conformacionales y grado de actividad biológica que la teoría postula para dar cuenta de los fenómenos.

\subsubsection{Los modelos efectivos de la teoría MWC}

Los modelos efectivos o actuales, elementos de M, son los sistemas que, además de satisfacer los axiomas impropios anteriores, satisfacen la(s) ley(es) fundamental(es) de la teoría, siendo así la contraparte modelo-teórica de tal(es) ley(es).

Definición 2. M(MWC): $\quad x=\left\langle O, S, A, I, T,\{\tau, r\}, \rho, \sigma, \beta, \delta, \zeta, \kappa_{S}, \kappa_{A}\right.$, $\left.\kappa_{I}\right\rangle$ es un sistema MWC ( $\left.x \in \mathbf{M}(\mathbf{M W C})\right)$ sii $x \in \mathbf{M}_{\mathrm{p}}(\mathbf{M W C}) \&$

(1) L1 $\forall t \in T \forall \forall \in O$ :

$$
\begin{aligned}
& \zeta(\sigma(o, t), t) \neq \zeta(\sigma(o, t+1), t+1) \text { sii } \forall j \sigma(o, t)_{j}=\langle\varnothing, \varnothing, \varnothing\rangle= \\
& \sigma(o, t+1)_{j}[1 \leq j \leq \rho(\mathrm{O})]
\end{aligned}
$$

$D f A u x$ 4: $\eta(o, @, t)=|\{o / \zeta(\langle\sigma(o, t), t\rangle)=@\}|$

(2) L2 $\forall t, t^{\prime} \in T \forall o \in O$ :

$$
\begin{aligned}
& \eta(o, r, t) \div \eta(o, \tau, t)=\eta\left(o, r, t^{\prime}\right) \div \eta\left(o, \tau, t^{\prime}\right) \text { sii } \forall j \sigma(o, t)_{j}= \\
& \langle\varnothing, \varnothing, \varnothing\rangle=\sigma\left(o, t^{\prime}\right)_{j}[1 \leq j \leq \rho(O)]
\end{aligned}
$$

DfAux 5: $i_{0 t}=[1 \leq j \leq \rho(O)]$

$$
\begin{aligned}
& \left|\left\{\mathrm{o} / \forall j \sigma(o, t)_{\dot{\mathrm{i}}}=\langle\varnothing, \varnothing, \varnothing\rangle \& \xi(\langle\sigma(o, t), t\rangle)=\tau\right\}\right| \div \mid\left\{\mathrm{o} / \forall j \sigma(o, t)_{j}\right. \\
& =\langle\varnothing, \varnothing, \varnothing\rangle \& \zeta(\langle\sigma(o, t), t\rangle)=r\} \mid
\end{aligned}
$$

DfAux 6: $\left|Y_{t}\right|=\mid\{x \in \Psi / x$ está unido a algún protómero de algún oligómero de $O$ en $t\} \mid$

DfAux 7: $\varepsilon_{\Psi @ t}=\left|\Psi_{t}\right| / \kappa_{\Psi @}$

(3) L3 $\forall t \in T$ :

$$
\delta(t)=\frac{i_{0} \frac{\left(1+\varepsilon_{I \tau t}\right)^{\rho_{0}}}{\left(1+\varepsilon_{A r t}\right)^{\rho_{0}}} \varepsilon_{S \tau t}\left(1+\varepsilon_{S \tau t}\right)^{\rho_{0}-1}+\left(\varepsilon_{S \tau t}\right)\left(1+\varepsilon_{S r t}\right)^{\rho_{0}-1}}{i_{0} \frac{\left(1+\varepsilon_{I \tau t}\right)^{\rho_{0}}}{\left(1+\varepsilon_{A r t}\right) \rho_{0}}\left(1+\varepsilon_{S \tau t}\right) \rho_{0}+\left(1+\varepsilon_{S r t}\right) \rho_{0}}
$$


El axioma (1), o ley de la transición alostérica, establece que si en un cierto momento un oligómero está en un cierto estado conformacional y su estado combinatorio es no ligado (esto es, todos los sitios de unión de sus protómeros están vacíos), entonces al instante siguiente, este oligómero cambia su estado conformacional si y solo si permanece no ligado. Esto es, si el oligómero se encuentra unido a un ligando, ya no cambia su estado conformacional, el ligado "fija" al oligómero en un estado conformacional determinado.

El segundo axioma (2), la segunda ley general, establece que la proporción de los oligómeros en cada estado conformacional permanece constante en dos momentos diferentes si y solo si todos los oligómeros permanecen no ligados. Para simplificar la notación abreviaremos "el número de oligómeros en estado conformacional @ en el momento $t$ ” como ' $\eta(o$, @, t)' (eso es, utilizaremos '@' como la variable de estado conformacional, de allí $D f 4$.

El tercer axioma (3), es la ley que conecta la actividad biológica con el estado conformación y el grado de unión. Esta ley establece que a cada momento el grado de actividad biológica coincide "cualitativamente" ${ }^{12}$ con la proporción de oligómeros unidos (en ambos estados conformacionales) sobre el total de oligómeros. Por mor de la exposición, hemos introducido las siguientes abreviaturas: $(D f 5)$ ' $\mathrm{o}_{0 t}$ ' para nombrar abreviadamente a la proporción entre oligómeros sin unión en los dos estados conformacionales en el instante $t$; $(D f 6)$ ' $\mid \Psi_{t}$ '’ para nombrar el número de lugares de unión del oligómero en la población unidos a ligando del tipo $\Psi$ en el momento $t:(D f 7)$ ' $\varepsilon_{\Psi @ t}$ ' par nombrara la "concentración de ligando normalizada", que se corresponde con el número de lugares de unión del oligómero en la población unidos a ligando del tipo Y en la conformación @ en el momento $t$ sobre la constante de disociación $\kappa$ para esa conformación y ese ligando.

Una vez identificados los modelos potenciales y los modelos actuales o efectivos, debemos determinar los modelos parciales, e.e. las partes de los modelos potenciales que no presuponen las leyes de la teoría, que expresan los conceptos con los que se describen los fenómenos a explicar.

${ }^{12}$ Conviene aquí recordar la frase de gran importancia escrita en el artículo original de presentación de la teoría: "Debemos discutir solamente las predicciones cualitativas más características del modelo en sus aplicaciones a los sistemas reales" (Monod, Wyman y Changeux, 1965, p.95), donde los autores explicitan que no habría posibilidad de predicción cuantitativa alguna debido a que dicho modelo predice un parámetro distinto, aunque analogable, al de actividad biológica. 


\subsubsection{Los modelos parciales de la teoría MWC}

Siguiendo el marco sneedeano-estructuralista, se identifican los sistemas empíricos que la teoría tiene por objeto dar cuenta, como aquellos fenómenos cuya identificación no necesita o presuponen las leyes teóricas o principios introducidos por la teoría. Para poder caracterizar esta clase de modelos es necesario establecer la distinción entre los conceptos T-teóricos y T-no teóricos de la teoría, es decir, entre aquellos conceptos que son propios, específicos o distintivos de la teoría en cuestión y aquellos que no lo son. Utilizaremos para ello la distinción habitual que aparece precisada en Balzer, Moulines \& Sneed (1987, pp. 73-74).

Dentro del grupo de los conceptos no-teóricos de MWC se encuentran: el conjunto de los oligómeros $(O)$, concepto perteneciente a la teoría (identificable, pero todavía no reconstruida de la bioquímica) que se ocupa de explicar la estructura de las proteínas; también es no teórico el conjunto de tipos de ligandos $(S, A, I)$, conceptos tomados de (alguna teoría, identificable, pero todavía no reconstruida), perteneciente a la química, por tanto su determinación es independiente de las leyes de la TMWC. Algo similar ocurre con la función protómeros $(\rho)$ concepto perteneciente a la teoría (identificable, pero todavía no reconstruida de la bioquímica) que se ocupa de explicar la estructura de los proteínas, con la función unión o binding $(\beta)$, que refiere justamente a la unión ligando-oligómeros, concepto tomado de (alguna teoría, identificable, pero todavía no reconstruida, perteneciente a) la química y con la función sucesión de uniones $(\sigma)$. Entre los conceptos no teóricos también se encuentra la función de actividad biológica $(\delta)$ que, una vez más, dispone en la bioquímica de distintos métodos de determinación, dependiendo del sistema bioquímico del que se trate, todos TMWC independientes. Por ejemplo en los sistemas enzimáticos la actividad catalítica (o velocidad catalítica) es la actividad biológica de estas proteínas y se cuantifica como la cantidad de sustancia transformada en el tiempo, mientras que en el caso de las proteínas transportadoras como la hemoglobina se cuantifica la actividad como porcentaje de saturación o cantidad de sitios ocupados. Finalmente el tiempo representado mediante un orden lineal isomórfico con el par $\langle\mathbf{N},<\rangle$, que proviene de la cronometría es también un concepto MWC no teórico.

Dentro del grupo de los conceptos teóricos de MWC se encuentran: los estados conformacionales representados por $\tau$ y $r$, y la función estado conformacional $(\zeta)$ introducida por primera vez por MWC. Recordemos que aquello que se puede determinar empíricamente (o independientemente de MWC) es un patrón de actividad biológica y no un estado conformacional, 
pues, que una proteína este en un estado conformacional ( $\tau$ o $r$ ) o que cambie su conformación constituyen hipótesis acerca de la estructura y cambio estructural de la proteína propios de la teoría MWC. Es la corrección de esas hipótesis la que se prueba con la aplicación de las leyes de la teoría ya que dichos estados concordarán con una actividad biológica dada. Por lo tanto la determinación de si una proteína oligomérica está en una conformación u otra sólo se puede establecer mediante las leyes de MWC. Si bien las constantes de disociación $\left(\kappa_{S}, \kappa_{A}, \kappa_{I}\right)$ están íntimamente relacionadas con la relación de unión $(\beta)$, pues expresan la tendencia que tiene un oligómero a unirse a un ligando particular (su "afinidad"), éstas no pueden determinarse independientemente de la previa determinación del estado conformacional del oligómero y, como ya se mencionó, esto sólo puede hacerse de manera dependiente de las leyes de MWC.

Los denominados modelos parciales $\mathbf{M}_{\mathrm{pp}}$, que describen, mediante conceptos no-teóricos relativos a la teoría en cuestión, los sistemas o fenómenos a los que se va a pretender aplicar teoría, constituyen pues la "base empírica" de la teoría.

Definición 3. $\mathbf{M}_{\mathrm{pp}}(\mathrm{MWC}): y=\langle O, S, A, I, T,\{\tau, r\}, \rho, \sigma, \beta, \delta\rangle$ es una sistema MWC parcial sii existe una $x$ tal que:

$$
x=\left\langle O, S, A, I, T,\{\tau, r\}, \rho, \sigma, \beta, \delta, \zeta, \kappa_{S}, \kappa_{A}, \kappa_{I}\right\rangle \mathrm{y} \mathrm{x} \in \mathbf{M}_{\mathrm{p}}(\mathrm{MWC})
$$

\subsection{Las aplicaciones propuestas y el elemento teórico básico de la teoría MWC}

El dominio de aplicaciones propuestas I constituye la clase de aquellos sistemas empíricos a los que uno desea aplicar la ley fundamental de la teoría. Ellos no pueden ser identificados por medios puramente formales. Lo único que podemos decir desde un punto de vista formal es que una aplicación propuesta es un modelo parcial, esto es, una parte de la realidad descrito/identificado con el vocabulario T-no teórico. En nuestro caso, esto significa que $\mathbf{I}(\mathbf{M W C}) \subseteq \mathbf{M}_{\mathrm{pp}}(\mathrm{MWC})$.

Los miembros de I(MWC), a los que uno desea aplicar las leyes fundamentales de la teoría MWN, son sistemas empíricos en donde ciertos oligómeros formados por un número $n$ de protómeros - que pueden unirse a cierta cantidad de ligandos, en un tiempo dado, mediante una unión quimica especifica-presentan cierta actividad biológica que evoluciona en el tiempo. Por ejemplo, las curvas de actividad catalíticas obtenidas al agregar a una población de enzimas desoxitimidina kinasa distintas cantidades de 
sustrato (ATP), tanto en presencia como ausencia de inhibidor (dCDP). Esta enzima es una molécula formada por cuatro protómeros, que cataliza la transformación química del nucleótido de timidita, en presencia de ATP, en timidita-5-fosfato (Monod et al. 1965, pp. 102).

El elemento teórico básico de la teoría alostérica, T(MWC), puede ahora ser identificado a través de los conjuntos determinados previamente:

$\mathrm{T}(\mathrm{MWC}):=\langle\mathrm{K}(\mathrm{MWC}), \mathrm{I}(\mathrm{MWC})\rangle$.

\section{La red teórica de la teoría MWC}

Se puede decir que salvo los casos más simples de teorías científicas, éstas deben ser consideradas como agregados de varios elementos teóricos. La noción estructuralista que captura esta complejidad es el de red teórica y representa la estructura (sincrónica) de una teoría en sus diferentes estratos, en sus diversos niveles de especificidad. La idea es la siguiente: en general en una red hay unas leyes fundamentales (en muchos casos una única) en la cúspide de la jerarquía, que se aplican a todos los diferentes tipos sistemas de que da cuenta la teoría, y además diferentes grupos de leyes "especiales", que se aplican cada uno sólo a algunos de los sistemas, con distintos grados de especialización en la base. La relación que se da entre los elementos teóricos más generales y los más específicos es el de especialización (relación no-deductiva). Mediante la relación de especialización los elementos más generales de la cúspide se van concretando progresivamente en diversas direcciones cada vez más restrictivas y específicas, las ramas de la red teórica (Díez \& Lorenzano 2002). Cada rama añade restricciones/leyes adicionales a las del elemento teórico básico general que hemos visto, restricciones adicionales ideadas para dar cuenta de aplicaciones específicas. Todas las aplicaciones de todas las ramas deben cumplir las leyes del elemento general y además las leyes específicas de su rama. Presentaremos a continuación los diferentes tipos de restricciones o leyes adicionales que caracterizan las diferentes ramas de la red teórica de MWC para las diferentes aplicaciones o fenómenos que quiere cada una explicar.

En particular, en nuestra teoría, las especializaciones consisten en la introducción de las siguientes restricciones:

(a) el tipo de interacción que tenga lugar entre los protómeros del oligómero, que puede ser de dos tipos: (i) interacción no cooperativa o de Michaelis-Menten; (ii) interacción cooperativa o alostérica propiamente dicha. 
(b) el tipo de ligando con que interactúan los oligómeros: (i) "efecto homotrópico" producto de la interacción (unión) con ligandos (sustratos) idénticos; (ii) "efecto heterotrópico no cooperativo" producto de la interacción (unión) con ligandos distintos, sustrato y activador; y (iii) "efecto heterotrópico cooperativo" producto de la interacción (unión) con ligandos distintos, sustrato e inhibidor.

\subsection{Primer nivel de especialización}

Según el tipo de interacción que tengan entre sí los protómeros del oligómero.

Especificación del tipo (ai): Especialización en oligómeros que no presentan cooperatividad entre subunidades y cuya actividad es no cooperativa o tipo Michaelis-Menten. La restricción aquí especifica que, las afinidades por los ligandos son iguales para ambos estados conformacionales y hay muchos menos oligómeros vacíos en estado $\tau$ que en el estado $r$.

Definición 4. $\mathbf{M}(\mathbf{M M}): x$ es un sistema $M W C$ no cooperativo o tipo Michaelis-Menten $(x \in \mathbf{M}(\mathbf{M M}))$ sii

(1) $x \in \mathbf{M}(\mathbf{M W C})$

(2) $\kappa_{S_{\tau}}=\kappa_{S_{r}} \& \forall t \in T[1 \leq j \leq \rho(O)]:$

$\left|\left\{o / \forall j \sigma(o, t)_{j}=\langle\varnothing, \varnothing, \varnothing\rangle \& \xi(\langle\sigma(o, t), t\rangle)=\tau\right\}\right| \ll \mid\left\{o / \forall j \sigma(o, t)_{j}=\right.$ $\langle\varnothing, \varnothing, \varnothing\rangle \& \xi(\langle\sigma(o, t), t\rangle)=\mathrm{r}\} \mid$

Con estas restricciones adicionales, la actividad biológica toma el siguiente valor específico:

$\forall x \in \mathbf{M}(\mathbf{M M}):$

$$
\delta(t)=\frac{\varepsilon_{S \tau t}}{S_{t}}
$$

Esta forma especializada que adopta la tercera ley fundamental de TMWC es la presentada en el artículo del '65 para los sistemas tipos Michaelis-Menten (Monod et al. 1965, p. 92). La forma de la curva dosisrespuesta que adopta este sistema se muestra en la figura 3.

Especificación del tipo (aii). Especialización en oligómeros cuyas subunidades presentan cooperatividad entre sí por lo que su actividad es cooperativa o tipo alostérica propiamente dicha. Aquí también hay menor cantidad de oligómeros vacíos en estado $\tau$ que en estado $r$, pero ahora la afinidad para los ligandos es mucho mayor para $r$ que para $\tau$.

Definición 5. $\mathbf{M}(\mathbf{A l}): x$ es un sistema $M W C$ cooperativo o tipo alostérico $(x \in \mathbf{M}(\mathbf{M M}))$ sii

(1) $x \in \mathbf{M}(\mathbf{M W C})$ 


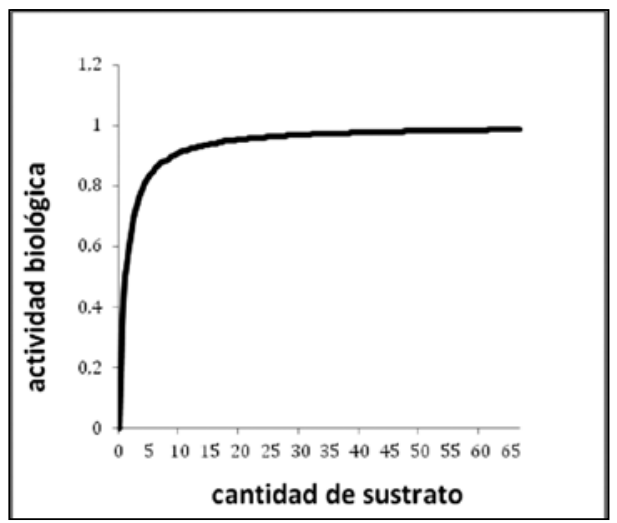

Fig. 3. En esta figura se muestra el perfil de la curva de actividad biológica en función de cantidad de sustrato para los sistemas tipo Michaelis-Menten. Notar la forma hiperbólica de la misma. La simulación se realizó para un $\mathrm{n}=4$, y una $\kappa_{\text {sr }}=1$ (unidades arbitrarias).

(2) $\kappa_{S \tau}<<\kappa_{S r} \& \forall t \in T[1 \leq j \leq \rho(O)]$ :

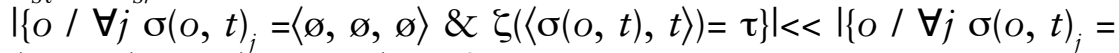
$\langle\varnothing, \varnothing, \varnothing\rangle \& \xi(\langle\sigma(o, t), t\rangle)=\mathrm{r}\} \mid$

Cuanto mayor es la diferencia entre las afinidades por el sustrato de uno y otro estado (siendo el más afín el R) más sigmoidea se vuelve la curva. El valor específico que adquiere la actividad a lo largo de la evolución es el siguiente, presentada en el artículo del '65 (Monod et al. 1965, p. 93):

$\forall x \in \mathbf{M}(\mathbf{A l}):$

$$
\delta(t)=\frac{\left(\varepsilon_{S \tau t}\right)\left(1+\varepsilon_{S r t}\right) \rho_{0}^{-1}}{\iota_{0} \frac{\left(1+\varepsilon_{I \tau t}\right)^{\rho_{0}}}{\left(1+\varepsilon_{A r t}\right)^{\rho_{0}}}\left(1+\varepsilon_{S \tau t}\right)^{\rho_{0}}+\left(1+\varepsilon_{S r t}\right) \rho_{0}}
$$

En el artículo del ' 65 los autores llaman "sistema $K$ "13 la interacción combinada del sustrato y al menos alguno de los tipos de efectores los cuales modifican aparentemente la afinidad de los oligómeros por el sustrato. En esta especialización, que es también terminal, se caracterizan los sistemas formados por oligómeros que reaccionan con sustrato, efector inhibidor y efector activador. Siguiendo la nomenclatura "heterotrópico" y "homotrópico" introducida por los autores (Monod et al. 1965, p. 89) a estos sistemas también podríamos llamarlos "heterotrópicos", pero en esta propuesta de reconstrucción utilizaremos los términos "heterotrópico" y "homotrópico" para nombrar las especializaciones que se corresponden al segundo nivel.

${ }^{13}$ Los autores también mencionan un "sistema $V$ " que no desarrollan dado que cuentan con mayor cantidad de ejemplos para el "sistema K" (Monod et. al. 1965, p.95). 


\subsection{Segundo nivel de especialización}

Según el tipo de ligando con que interaccione el oligómero.

Los sistemas alostéricos (aii) puede satisfacer restricciones adicionales, dependiendo de si el sustrato es el único ligando que se une al oligómero, o si se une al oligómero también activadores o inhibidores.

En la primera subespecialización (bi), que cumple con la restricción introducida en (aii), se añade la constricción que corresponde al "efecto homotrópico", es decir, que todo los protómeros del oligómero solo se unen a ligando sustrato o, lo que es lo mismo, que el sistema carece de ligandos reguladores.

Definición 6:

$\mathbf{M}(\mathrm{Ho}): x$ es un sistema $M W C$ del tipo alosterico con "efecto bomotrópico" $(x \in \mathbf{M}(\mathrm{Ho}))$ sii

(1) $x \in \mathbf{M}(\mathbf{A l})$

(2) $\forall o \in O, \forall t \in T[1 \leq j \leq \rho(O)]: \forall j \sigma(o, t)_{j}=\langle s, \varnothing, \varnothing\rangle$

Con estas restricciones adicionales, la actividad biológica toma el siguiente valor específico:

$\forall x \in \mathbf{M}(\mathrm{Ho}):$

$$
\delta(t)=\frac{\left(\varepsilon_{S \tau t}\right)\left(1+\varepsilon_{S r t}\right)^{\rho_{0}-1}}{i_{0}+\left(1+\varepsilon_{S r t}\right)^{\rho_{0}}}
$$

Un caso de sistema homotrópico es hemoglobina-oxígeno. El perfil que adopta la curva dosis-respuesta de un sistema homotrópico se muestra en la figura 4.

En la segunda subespecialización (bii), que cumple con la restricción introducida en (aii), se añade la constricción que corresponde al efecto "heterotrópico no cooperativo", esto es que todos los protómeros de todos los oligómeros se unen, además de al sustrato, a los activadores.

\section{Definición 7:}

$\mathbf{M}($ Henc): $x$ es un sistema $M W C$ del tipo alosterico con "efecto beterotrópico no cooperativo $(x \in \mathbf{M}(\mathrm{Henc}))$ sii

(1) $x \in \mathbf{M}(\mathbf{A l})$

(2) $\forall o \in O, \forall t \in T[1 \leq j \leq \rho(O)]: \forall j \sigma(o, t)_{j}=\langle s, a, i\rangle$ con $a \neq \varnothing \& i=\varnothing$

Cuando el efector es un activador el sistema es llamado por los autores " $K$ positivo". En este caso se da una interacción simultánea entre el oligómero, el ligando sustrato y el ligando activador que conlleva a que la actividad pierda la forma sigmoidea y se transforme en hipérbola con una aparente pérdida de cooperatividad entre subunidades o protómeros. Por 
ello lo denominamos "efecto heterotrópico no cooperativo". Se considera que todos los sitios para tal efector se unen al activador. Un caso de este tipo de efecto lo representa la enzima PKF en presencia de AMP. El valor específico que adquiere la actividad a lo largo de la evolución es el siguiente:

$\forall x \in \mathbf{M}($ Henc):

$$
\delta(t)=\frac{\left(\varepsilon_{S \tau t}\right)\left(1+\varepsilon_{S r t}\right)^{\rho_{0}-1}}{\iota_{0} \frac{1}{\left(1+\varepsilon_{A r t}\right)^{\rho_{0}}}+\left(1+\varepsilon_{S r t}\right) \rho_{0}}
$$

El perfil que adopta la curva dosis-respuesta de un sistema heterotrópico no cooperativo se muestra en la figura 4.

En la tercera subespecialización (biii), que cumple con la restricción introducida en (aii), se añade la constricción que corresponde al efecto "heterotrópico cooperativo", esto es, cuando los protómeros de los oligómeros se unen, a demás del sustrato, a los inhibidores.

Definición 8:

$\mathbf{M}(\mathrm{Hec}): x$ es un sistema $M W C$ del tipo alosterico con "efecto heterotrópico cooperativo $(x \in \mathbf{M}(\mathrm{Hec}))$ sii

(1) $x \in \mathbf{M}(\mathbf{A l})$

(2) $\forall o \in O, \forall t \in T[1 \leq j \leq \rho(O)]: \forall j \sigma(o, \forall t)_{j}=\langle s, a, i\rangle$ con $i \neq \varnothing \& a=\varnothing$

En el artículo del ' 65 los autores llaman "sistema K negativo" a los sistemas formados por oligómeros que reaccionan con sustrato y efector inhibidor de manera tal que su actividad sigue presentando la forma sigmoidea pero más acentuada por el efecto del inhibidor. Por ello lo denominamos efecto "heterotrópico cooperativo". Se considera que todos los sitios para tal efector se unen al inhibidor. Dos ejemplos de este tipo de efecto son la enzima PKF cuando está en presencia de citrato y la desoxitimidina quinasa en presencia de dCDP. El valor específico que adquiere la actividad a lo largo de la evolución es el siguiente:

$\forall x \in \mathbf{M}(\mathrm{Hec}):$

$$
\delta(t)=\frac{\left(\varepsilon_{S \tau t}\right)\left(1+\varepsilon_{S r t}\right)^{\rho_{0}-1}}{i_{0}\left(1+\varepsilon_{I \tau t}\right)^{\rho_{0}}+\left(1+\varepsilon_{S r t}\right)^{\rho_{0}}}
$$

El perfil que adopta la curva dosis-respuesta de un sistema heterotrópico cooperativo se muestra en la figura 4. 


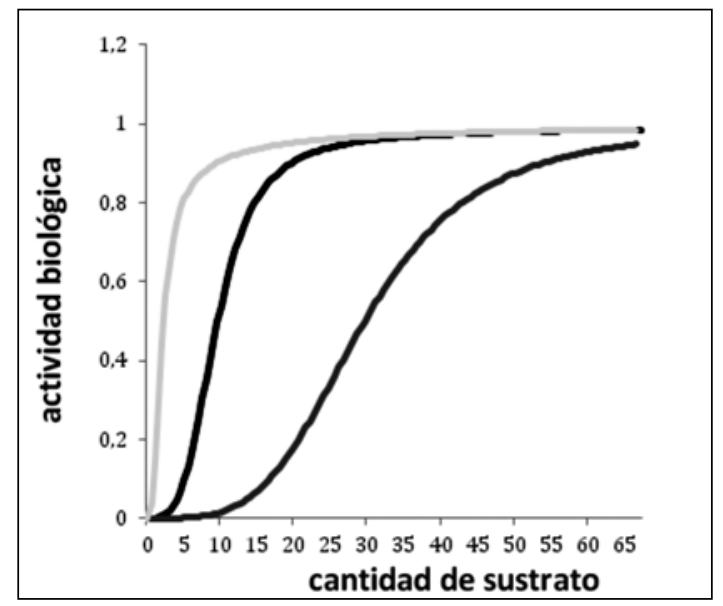

Fig. 4. En la figura se muestra con línea gris clara la curva que se obtiene para el efecto heterotrópico no cooperativo (Henc), en línea negra el perfil de la curva del efecto homotrópico (Ho) y en línea gris oscura el perfil correspondiente al efecto heterotrópico cooperativo (Hec). Para las simulaciones mostradas se consideraron los siguiente valores: $t_{0}=1000, \rho(o)$ $=4, \kappa_{s \mathrm{r}}=1, \kappa_{i \tau}=1$ (para Hec), $\kappa_{a r}=1$ (para Henc), en todos los casos con unidades arbitrarias.

La estructura de la red teórica de la teoría MWC se refleja en el siguiente grafo:

$1^{\mathrm{o}}$ N.E.

$\mathrm{M}(\mathrm{MM})$

$\mathrm{M}(\mathrm{AI})$

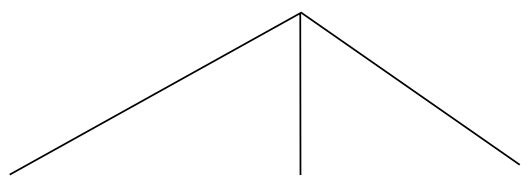

$2^{\circ}$ N.E.

$\mathrm{M}(\mathrm{Ho})$

$\mathbf{M}($ Henc)

$\mathbf{M}(\mathrm{Hec})$

Fig. 5 Representación de la red teórica de la teoría MWC presentada en 1965 


\section{Conclusión}

Hemos presentado aquí los primeros pasos para la reconstrucción de una parte de la bioquímica, aquella que da cuenta de la actividad biológica de las proteínas, realizada con las herramientas proporcionadas por la concepción estructuralista de las teorías. Ésta constituye hasta ahora la única reconstrucción (semanticista o no) de la teoría MWC. Como muestra la reconstrucción, la finalidad de esta teoría es explicar los diferentes patrones de evolución de actividad biológica de poblaciones de oligómeros según su diferente unión con ligandos.

En esta propuesta reconstructiva se hacen explícitos: (i) el aparato conceptual de la teoría; (i) las entidades teóricas que la teoría postula para explicar la evolución de actividad, (ii) las leyes generales que se aplican a todos los sistemas con diferentes evoluciones, y (iv) las leyes especiales que satisfacen cada uno de los tipos de sistemas de las diferentes evoluciones. Esta reconstrucción realiza pues un aporte a la comprensión metateórica de esta teoría y del "alosterismo" al explicitar por primera vez todos estos elementos.

Es pertinente aclarar que la elección de dónde tomar la teoría para su reconstrucción, esto es que fuera realizada principalmente a partir de los trabajos científicos originales y no de los libros de texto de bioquímica, se debió a que si bien en todos estos libros se encuentra el modelo MWC, la presentación que los mismos hacen diverge, dependiendo el libro, en mayor o menor medida de la presentación original. Las causas de esta divergencia aún no nos son del todo claras, pero sin duda son factibles de ser analizadas a partir de ésta reconstrucción, discusión que dejamos para más adelante. Quedan también para trabajos futuros el análisis y explicitación de algunos elementos aquí no tratados como las restricciones cruzadas y las condiciones de ligadura, además del análisis de otras teorías diferentes que se presentaron como alternativa a la explicación de los mismos, o similares, fenómenos.

\section{Referencias bibliográficas}

Balzer, W.; Moulines, C. U.; Sneed, J. D. (1987) An Architecture for Science. The Structuralist Program. Dordrecht: Reidel.

Bindslev N. (2008) Drug-Acceptor Interactions: Modeling theoretical tools to test and evaluate experimental equilibrium effects. Co-Action Publishing. 
Cantor, Ch. R.; Schimmel P. R. (1980) The behavior of biological macromolecules (part III). New York: W.H. Freeman and company.

Cartwright, N. (2008) "Reply to Ulrich Gähde.”, in: Bovens, Luc; Hoefer, Carl; Hartmann, Stephan (eds.). Nancy Cartwright's Philosophy of Science. New York: Routledge, 2008, pp. 65-66.

Darden, L.; Maull, N. (1977) "Interfield Theories". Philosophy of Science, 44 pp. 43-64.

Díez, J. A.; Lorenzano, P. (2002) "La concepción estructuralista en el contexto de la filosofía de la ciencia del siglo XX", in: Diez, J. A.; Lorenzano, P. (eds.). Desarrollos actuales de la metateoría estructuralista: problemas $y$ discusiones. Quilmes: Universidad Nacional de Quilmes/Universidad Autónoma de Zacatecas/Universidad Rovira i Virgili, 2002, pp. 13-78.

Koshland, D.E.; Nemethy JR.; Filmer G. D. (1966) "Comparison of experimental binding data and theoretical models in proteins containing subunits". Biochemistry 5(1):365-368.

Monod, J.; Changeux, J.-P.; Jacob, F. (1965) “Allosteric proteins and cellular control systems". Journal of Molecular Biology 6: 306-320.

Monod, J.; Wyman, J.; Changeux, J.-P. (1965) "On the nature of allosteric transitions: a plausible model". Journal of Molecular Biology. 12: 88118.

Monod, J. (1965) "From enzymatic adaptation to allosteric transitions", Nobel Lecture. 